\title{
Improving patient safety through an emergency call safety huddle
}

\author{
Authors: Shuaib Quraishi and Claire Rowley
}

\section{Introduction}

Communication among members of the emergency team is integrally linked to patient safety. ${ }^{1}$ The need to promptly identify and manage the acutely unwell patients is key towards preventing harm to patients. A short daily meeting can help save lives by helping emergency teams to work together more effectively. At Surrey and Sussex NHS Trust (SASH) we have approximately five to eight emergency calls in a 24 -hour period. These are composed of medical emergency team (MET), which is composed of the medical registrar, senior house officer, foundation doctor and a critical care outreach nurse (CCOT). A cardiac arrest team is composed of the MET team as well as an anaesthetist. In the past the emergency team would initially meet over an unwell patient unaware of who each other was, what role they played and what was expected of them. It is known that there is a 1 in 400 million chance of the same team working together again. ${ }^{2}$

The Safety Huddle has been a part of the culture of improving patient care at SASH since October 2016. Members of the cardiac arrest and medical emergency teams meet each other, roles are allocated every morning and learning from previous emergencies is discussed. Roles and training needs are documented through a standardised checklist on a daily basis. This is in order to create effective teamworking and improve patient safety.

\section{Methods}

At SASH we wanted to elicit whether the safety huddles were actually serving their purpose. A qualitative survey was sent via SurveyMonkey to medical and nursing staff who had attended the safety huddles. We had 29 responses from Nurses (CCU and CCOT), medical registrars, and junior doctors (SHO and foundation). A thematic analysis of free text comments was undertaken and the following themes were identified.

\section{Results}

> Structure of the team. $100 \%$ of respondents found the huddle to be useful. It identified and allocated roles and created familiarity between team members

> Improve team working. $100 \%$ felt the huddle improved team working.
> Patient safety. $91 \%$ of respondents felt patient safety was improved. This is through increased efficiency during emergencies, effective team working, better organisation and early involvement with critical care.

> Identification of learning needs. $87 \%$ felt learning needs that were identified at the safety huddle had been addressed.

The questionnaire also asked where improvements could be made and these were as follows.

> Night safety huddle. $72 \%$ would like to introduce an emergency huddle for the night team.

> Debriefing. A debrief session would be useful for feedback on learning from emergencies.

\section{Conclusion}

Our MET audit for 2018 has demonstrated that we have made an improvement in patient outcomes by an increase in patients who made an immediate improvement (79\% in 2018 from $61 \%$ in 2017) versus the patients who made no improvement immediately post MET call ( $4 \%$ in $2018,21 \%$ in 2017 ).

This suggests that by implementing the safety huddle we may be working more effectively as a team, resulting in improved patient outcomes.

\section{References}

1 Brady PW, Muething S, Kotagal U et al. Improving situation awareness to reduce unrecognized clinical deterioration and serious safety events. Pediatrics 2013;131:e298-308.

2 Kent Surrey Sussex Academic Health Sciences Network. Patient Safety Collaborative. Implementation of the 10-minute meeting: a user's guide. Brighton and Sussex University Hospitals NHS Trust. 